\title{
Elektronische Krankengeschichte in der Schweizer Grundversorgung - Diskrepanz zwischen Anspruch und Wirklichkeit
}

Djalali, Sima ; Bhend, Heinz ; Rosemann, Thomas

\begin{abstract}
The most recent data on the use of electronic medical records (EMR) in Swiss practices are from 2007. Since then, software products have evolved. The aim of our article is to update the knowledge about the current status of EMR implementation in Swiss practices and to gain information about Swiss primary healthcare providers' views of software tools providing clinical decision support.
\end{abstract}

DOI: https://doi.org/10.1024/1661-8157/a001290

Posted at the Zurich Open Repository and Archive, University of Zurich ZORA URL: https://doi.org/10.5167/uzh-78328

Journal Article

Published Version

Originally published at:

Djalali, Sima; Bhend, Heinz; Rosemann, Thomas (2013). Elektronische Krankengeschichte in der Schweizer Grundversorgung - Diskrepanz zwischen Anspruch und Wirklichkeit. Praxis, 102(11):641-646.

DOI: https://doi.org/10.1024/1661-8157/a001290 
Djalali S, Bhend H, Rosemann T: Praxis 2013; 102(11): 641-646.

\title{
Elektronische Krankengeschichte in der Schweizer Grundversorgung - Diskrepanz zwischen Anspruch und Wirklichkeit
}

\author{
Sima Djalali, Heinz Bhend, Thomas Rosemann
}

\section{Hintergrund}

Bereits 2007 hat der Bund eine «eHealth Strategie Schweiz» verabschiedet. Darin wird eHealth als der «[integrierte] Einsatz von Informations- und Kommunikationstechnologien (IKT) zur Gestaltung, Unterstützung und Vernetzung aller Prozesse und Teilnehmerinnen und Teilnehmer im Gesundheitswesen» definiert [1].

Das Ziel ist demgemäss ambitioniert. Die Realität zeigt sich indes weit entfernt von einem flächendeckenden Einsatz von Informations- oder

Kommunikationstechnologien im Gesundheitswesen oder gar einer Vernetzung der einzelnen Akteure. Von Hausärzte Schweiz wurde wiederholt moniert, dass zuerst medizinische Daten digital in den Primärsystemen der Arztpraxen vorhanden sein müssen, bevor diese Daten in einem sogenannten elektronischen Patientendossier (EPD) gespeichert werden können [2]. Indes ist die Bereitschaft von niedergelassen Ärzten, medizinische Daten digital zu dokumentieren und auf eine elektronische Krankengeschichte (eKG; electronic medical record, EMR) umzustellen, gering ausgeprägt.

Eine Erhebung unter 1200 niedergelassen Ärzten in der Schweiz im Jahr 2007 zeigte, dass erst $11,7 \%$ aller befragten Ärzte und nur 8,9\% der Hausärzte mit einer vollelektronischen Krankengeschichte arbeiten. Interessanterweise gaben in dieser Befragung 66,9\% aller Ärzte an, sich auch in den nächsten drei Jahren keine eKG anschaffen zu wollen [3].

\section{Ziel dieser Arbeit}

In der hier präsentierten Arbeit werden die Ergebnisse einer Querschnittserhebung unter Schweizer HausärztInnen im Jahr 2012 analysiert. Das Ziel ist es, die Datenlage zu aktualisieren und Informationen über den Stand der Implementierung von elektronischen Krankengeschichten in der Schweizer Grundversorgung zu erhalten. Darüber hinaus wird die Zufriedenheit der Schweizer HausärztInnen mit den am Markt erhältlichen Softwareprodukten untersucht sowie die Frage, welchen potenziellen Zusatznutzen Schweizer HausärztInnen in elektronischen Krankengeschichten sehen, der über die papierlose Dokumentation von medizinisch relevanten Informationen hinaus geht (z.B. Unterstützung bei klinischen Entscheiden, Clinical Decision Support).

\section{Methodik}

Im Januar/Februar 2012 wurden aus dem Adressdatensatz von Hausärzte Schweiz 3913 Hausärztinnen und Hausärzte in der deutsch- und französischsprachigen Schweiz via Mailversand eingeladen, an der Umfrage teilzunehmen und erhielten den Link zu einem Online-Fragebogen.

Der Online-Fragebogen war den Teilnehmern jeweils während einem Monat nach Erhalt der Einladungen auf Deutsch und Französisch zugänglich. Ein Erinnerunsmail zur Teilnahme wurde in diesem Zeitraum nicht versandt.

Die Resultate der Befragung konnten zur Auswertung direkt aus der Datenbank des Online-Fragebogens in Microsoft Excel 2012 übernommen werden. 
Der Online-Fragebogen 2012 umfasste sieben items, davon vier Fragen mit EinfachAntwortmöglichkeit (Radio-Button), eine Frage mit mehrfacher Antwortmöglichkeit (Checkboxes) sowie zwei Fragen mit Freitextantwort.

Ausgewertet wurden nur die Fragen mit Einfach- resp. mehrfacher

Antwortmöglichkeit.

\section{Resultate}

3913 Einladungen wurden per E-Mail versendet. Im Zeitraum vom 21.01.2012 bis 28.02.2012 wurde der Online-Fragebogen 890 Mal beantwortet, 710 Mal auf Deutsch und 180 Mal auf Französisch. Dies entspricht einem Rücklauf von 22,7\%.

\section{Umstellungsprozess}

Die Frage zur Umstellung auf vollelektronische Krankengeschichten wurde von 859 Teilnehmern beantwortet.

Abbildung 1 zeigt die Verteilung der Antworten. Rund die Hälfte (50,6\%) der antwortenden Hausärzte hatte die Umstellung auf die elektronische Krankengeschichte bereits vollzogen $(37,1 \%)$ bzw. war zum Zeitpunkt der Erhebung im Umstellungsprozess begriffen (13,5\%).

Die andere Hälfte der Teilnehmer arbeitete bislang noch gar nicht mit elektronischen Krankengeschichten. Davon gaben $13,6 \%$ an, die Einführung immerhin zu erwägen, $8,5 \%$ innerhalb der nächsten 1-3 Jahre, 5,1\% mit einem Zeithorizont von mehr als drei Jahren. 35,7\% gaben an, die Umstellung überhaupt nicht in Erwägung zu ziehen.

\section{2: Wo stehen Sie im Umstellungsprozess (von Papier- Dokumentation zur elektronischen Krankengeschichte)?}
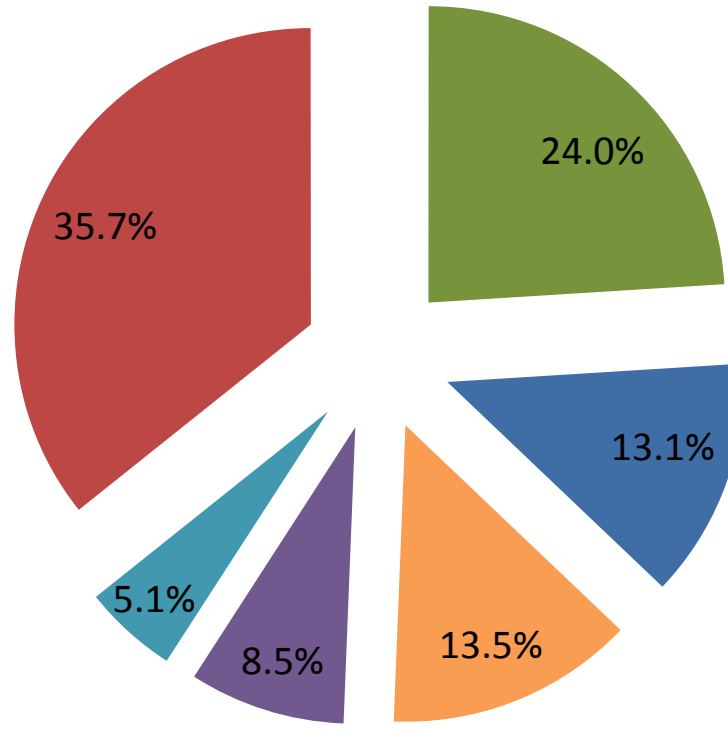

Ich habe schon umgestellt (>3 Jahre)

- Ich habe schon umgestellt (1-3 Jahre)

Ich bin aktuell am umstellen (+/- 1 Jahr)

- Ich plane die Umstellung in den nächsten 1-3 Jahren

- Ich plane die Umstellung später (>3 Jahre)

- Umstellung ist für mich kein

$13.5 \%$ Thema

$n=859$

Abb. 1: Umfrageergebnisse zum Umstellungsprozess 


\section{Zufriedenheit mit der aktuellen Krankengeschichte}

Die Frage zur Zufriedenheit mit der eigenen aktuellen Arbeitsweise (papierbasierte resp. elektronische Krankengeschichte) wurde von 858 Teilnehmern beantwortet. Die Teilnehmer waren gefordert, ihre Zufriedenheit nach folgender Klassifikation anzugeben: (a) «sehr zufrieden»: Bedürfnisse sind zu >80\% erfüllt; (b) «mässig zufrieden»: Bedürfnisse sind zu 51-80\% erfüllt; (c) «weniger zufrieden»: Bedürfnisse sind zu 35-50\% erfüllt; (d) «unzufrieden»: Bedürfnisse sind zu <35\% erfüllt.

Insgesamt gaben 447 Teilnehmer an, die Krankengeschichten ihrer Patienten papierbasiert zu führen. Davon waren 263 (30,7\% aller Antworten) mit dieser Arbeitsweise «sehr zufrieden». 148 (17,2\% aller Antworten) waren «mässig zufrieden», 32 (3,7\% aller Antworten) waren «weniger zufrieden» und 4 (0,5\% aller Antworten) waren «unzufrieden».

411 gaben an, eine elektronische Krankengeschichte zu führen. Die Mehrheit, 231 Teilnehmer (26,9\% aller Antworten), war mit der elektronischen Krankengeschichte ihres Softwareanbieters "sehr zufrieden», 139 (16,2\% aller Antworten) waren «mässig zufrieden», 34 (4\% aller Antworten) waren «weniger zufrieden» und 7 (0,8\% aller Antworten) waren «unzufrieden».

\section{Potenzial der elektronischen Krankengeschichte}

Auf die Frage, wie die HausärztInnen das funktionelle Potenzial der elektronischen Krankengeschichte (eKG) einschätzen im Verhältnis zur Umsetzung in den heute am Markt vertretenen Softwareprodukten, antworteten insgesamt 811 Teilnehmer, wobei die Mehrheit, 286 (35,2\% aller Antworten) angab, diese Einschätzung nicht machen zu können.

Dagegen hielt nur die Minderheit, 33 Teilnehmer (4\% aller Antworten), das funktionelle Potenzial der eKG bereits zu >90\% ausgeschöpft. 294 Teilnehmer (36,2\% aller Antworten) hielten die Ausschöpfung für mässig (40-74\% des Potenzials) bis schlecht ( $<40 \%$ des Potenzials).

\section{Bedarf an IT-Funktionen für Clinical Decision Support}

827 Teilnehmer beantworteten die Frage, wie gross ihr Bedarf nach Clinical Decision Support (CDS) sei. Als Definition für CDS galten «computerbasierte Hilfsmittel, welche die klinische Entscheidungsfindung durch Bereitstellung von Informationen zum Zeitpunkt der Entscheidung erleichtern, z.B.

Medikamenteninteraktionsprüfungen, Unverträglichkeitswarnungen, Hinweise auf fällige Laboruntersuchungen».

Tabelle 1 zeigt die Verteilung der Antworten. Danach beurteilte die überwiegende Mehrheit 702 Teilnehmer (84,9\% aller Antworten) CDS als hilfreich. 52 Teilnehmer (6,2\% aller Antworten) gaben an, keinen grösseren Bedarf an CDS zu sehen und 73 Teilnehmer (8,8\% aller Antworten) gaben an, keine Einschätzung machen zu können.

\begin{tabular}{|l|l|l|}
\hline Antwort: Clinical Decision Support... & Anzahl & Prozent $(\mathrm{n}=827)$ \\
\hline ...erachte ich als nicht nötig & 21 & $2,5 \%$ \\
\hline ...erachte ich als wenig hilfreich & 31 & $3,7 \%$ \\
\hline ...erachte ich als möglicherweise hilfreich & 311 & $37,6 \%$ \\
\hline ...erachte ich als sehr hilfreich & 391 & $47,3 \%$ \\
\hline ...weiss nicht/kann keine Angaben machen & 73 & $8,8 \%$ \\
\hline
\end{tabular}

Tab. 1: Umfrageergebnisse zum Bedarf an Clinical-Decision-Support-Funktionen 
Im Folgenden wählten die Teilnehmer aus einer vorgegebenen Auswahl von zehn spezifischen Clinical-Decision-Support-Funktionen diejenigen aus, die sie als praxisrelevant ansahen. Mehrfachantworten waren möglich.

Abbildung 2 zeigt die Häufigkeit der Antworten. Am häufigsten (738 Teilnehmer) wurden Softwarefunktionen als praxisrelevant bewertet, welche die Medikamentenpläne der Patienten auf Arzneimittelinteraktionen hin prüfen. Ein ähnlicher Prüfmechanismus, der die individuellen Medikamentenpläne mit dem Allergie- und Unverträglichkeitsverzeichnis des Patienten abgleicht, wurde vergleichbar häufig als praxisrelevant eingestuft (670 Teilnehmer).

Mehr als die Hälfte aller Teilnehmer gab an, Softwares für praxisrelevant zu erachten, die Warnhinweise geben, wenn Medikamente in kritischer Konstellation zu aktuellen Laborwerten stehen oder bestimmte Untersuchungen ausstehen (je >445 Teilnehmer). Zudem hielten sie Verlinkungen zwischen der eKG und klinischer Literatur und Guidelines für sinnvoll.

Hinweise auf Risikofaktorkonstellationen individueller Patienten und die Verlinkung mit Patienteninformationsmaterialien waren dagegen in den Augen von weniger Teilnehmern relevant (365 bzw. 309). Am wenigsten Anklang fand die Idee, DiätInformationen für Patienten im eKG-System zu hinterlegen (222 Teilnehmer).

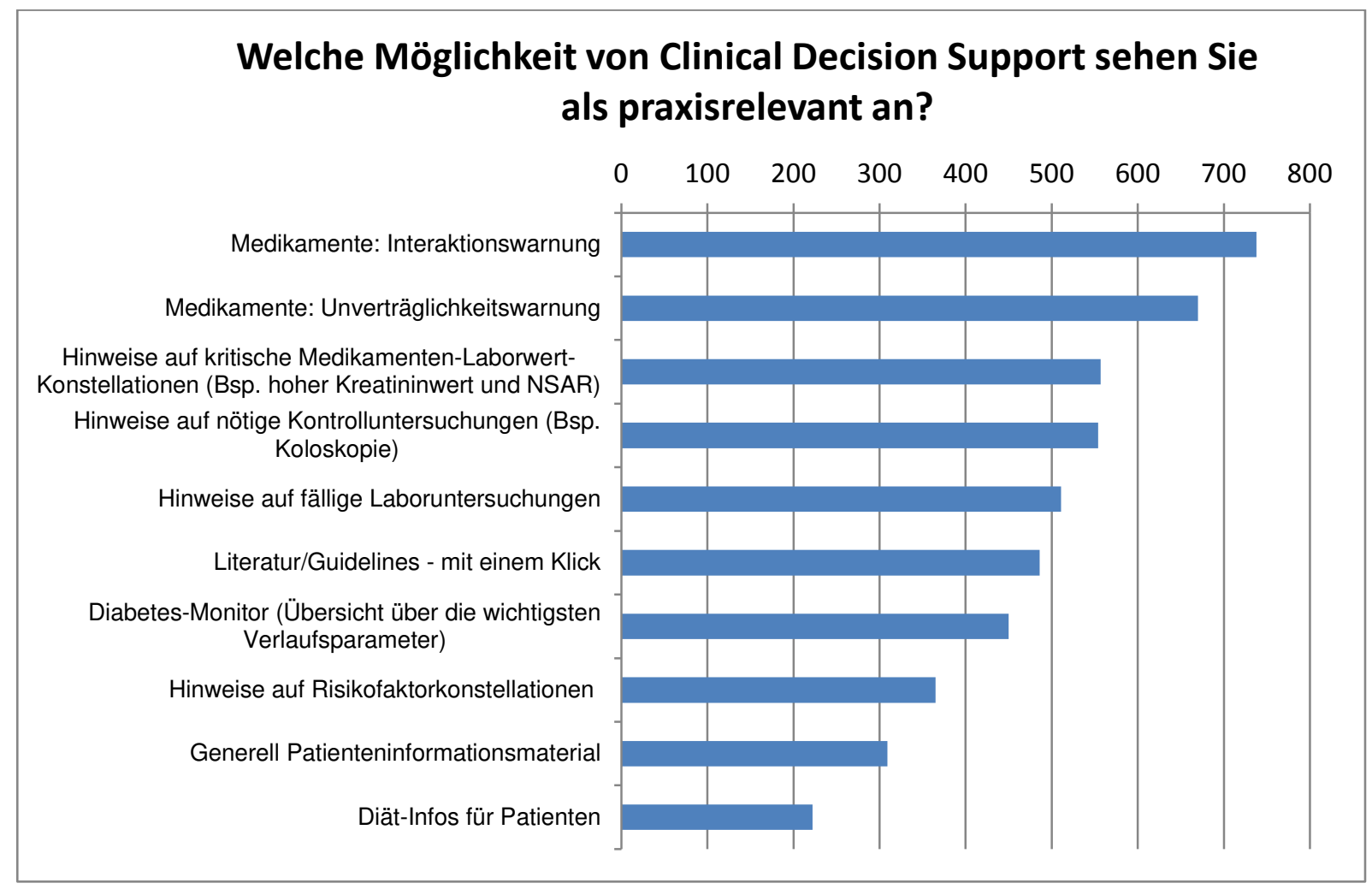

Abb. 2: Praxisrelevante Clinical-Decision-Support-Funktionen 
Eine Subgruppenanalyse der Ergebnisse von deutschsprachigen resp. französischsprachigen Teilnehmern wurde wegen der kleinen Fallzahlen nicht vorgenommen.

\section{Diskussion}

Ziel dieser Querschnittserhebung war es, Informationen über den aktuellen Stand der Implementierung von elektronischen Krankengeschichten (eKG) in der Schweizer Grundversorgung zu erhalten, sowie eine Einschätzung davon, welche Ansprüche Hausärztinnen und Hausärzte an eKG-Software stellen.

Wie sich zeigte, lag der Anteil der befragten HausärztInnen, die bereits auf eine elektronische Krankengeschichte umgestellt haben resp. zum Zeitpunkt der Befragung im Umstellungsprozess begriffen waren, bei 50,6\%. 13,6\% gaben an, die Umstellung zu planen und $35,7 \%$ gaben an, eine Umstellung nicht vorzusehen.

Verglichen mit früher publizierten Erhebungen, wonach erst 8,9\% der Hausärzte mit elektronischer Krankengeschichte arbeiten [3], scheint die Implementierung der elektronischen Krankengeschichte in der Schweizer Grundversorgung vorangeschritten zu sein.

Gegenüber einer 2010 durchgeführten Online-Umfrage unter 1031 Schweizer HausärztInnen, wovon 951 auf die Frage nach dem Umstellungsprozess in ihrer Praxis antworteten, scheint die Implementierung der elektronischen Krankengeschichte ebenfalls zugenommen zu haben. In dieser Umfrage gaben erst $40,5 \%$ der Teilnehmer an, bereits eine elektronische Krankengeschichte zu führen resp. innert desselben Jahres zu beginnen und 51,8\% gaben an, keine Umstellung vorzusehen [7].

Gegenüber einer weiteren, 2011 durchgeführten, Online-Umfrage unter 1058 Schweizer HausärztInnen, wovon 1047 auf die Frage nach dem Umstellungsprozess in ihrer Praxis antworteten, scheint die Implementierung der elektronischen Krankengeschichte dagegen zu stagnieren. In der Umfrage von 2011 lag der Anteil der Teilnehmer, die bereits eine elektronische Krankengeschichte führten resp. innert des nächsten Jahres planten, bei $51,2 \%$ und 39,8\% der Befragten gaben an, die Umstellung nicht vorzusehen [8].

Die Vergleiche mit früheren Erhebungen sind allerdings sehr kritisch zu sehen, weil die Fragebogen-items in den verschiedenen Erhebungen unterschiedlich waren und nichts über die Charakteristika der unterschiedlichen Teilnehmerpopulationen bekannt ist. Um valide Aussagen über den Zeitverlauf der Implementierung zu generieren, sind longitudinale Studien in einer für die Ärzte der Schweizer Grundversorgung repräsentativen Population unabdingbar.

Für sich gesehen, zeigen die Ergebnisse der hier vorliegenden Arbeit, dass die Implementierung von elektronischen Krankengeschichten in Schweizer Hausarztpraxen noch nicht sehr weit fortgeschritten ist. Um von einer flächendeckenden Implementierung sprechen zu können, die Grundlage für einen effizienten elektronischen Datenaustausch unter den Leistungserbringern ist, müssten weit mehr als 50\% den Technologieumstieg auf ein oder mehrere miteinander kompatible eKG-Systeme vollziehen, weil sonst ein zu grosser - für das 
Gesundheitssystem relevanter - Anteil von Leistungsbringern vom routinemässigen Datenaustausch ausgeschlossen bliebe.

Im Hinblick auf die ambitionierten Ziele der Schweizer eHealth-Strategie des Bundes, die immerhin bereits 2007 lanciert wurde, sind die Ergebnisse als ernüchternd zu betrachten und man darf eine erhebliche Diskrepanz zwischen Anspruch und Wirklichkeit postulieren.

Somit ist die erste wichtige Botschaft dieser Ergebnisse die Tatsache, dass die Politik gefordert scheint, die eigentlichen Anwender der elektronischen

Krankengeschichten verstärkt in die Realisierung der eHealth-Strategie einzubinden und gezielt nach den Gründen dafür zu suchen, die eine weitere Implementierung der elektronischen Krankengeschichte bisher verhindert haben.

Valide Daten über die Hinderungsgründe der Ärzte existieren aus dem Schweizer Kontext nicht. Hohe Kosten und ein in der täglichen Praxis nicht erlebter Mehrwert einer elektronischen Krankengeschichte dürfen aber wohl als entscheidende Faktoren unterstellt werden.

Immerhin gab die Mehrheit der HausärztInnen in der hier vorliegenden Befragung an, noch grosses Potenzial im Ausbau der eKG-Funktionalitäten zu sehen und erachtete speziell Clinical-Decision-Support-Funktionen als wünschenswerte Zusatzfunktion der eKG-Software.

Die Mehrheit der ÄrztInnen, die bereits mit einer eKG arbeiteten, gab zwar an, mit ihrem Softwareprodukt grösstenteils zufrieden zu sein. Demgegenüber muss aber der grosse Anteil ÄrztInnen gesehen werden, der angab, noch papierbasiert zu arbeiten und mit dieser Arbeitsweise absolut zufrieden zu sein. Diese ÄrztInnen benötigen stärkere Anreize als sie die Funktionen der heutigen eKGSoftwareprodukte bieten können, um von einer Umstellung auf elektronisches Arbeiten überzeugt zu werden.

Ein erster Schritt wäre, sich international zu orientieren und zu eruieren was in Gesundheitssystemen, die eine flächendeckende Verbreitung von elektronischen Krankengeschichten in der Grundversorgung aufweisen, etwa die Niederlande oder England, anders gemacht wurde.

In England etwa fiel die Implementierung der elektronischen Krankengeschichte in den Arztpraxen der Grundversorgung mit einer Honorarreform im Rahmen des Quality and Outcomes Frameworks (QOF) im Jahr 2004 zusammen [4]. Mit Einführung des QOF hatten Ärzte, die dem National Health Service (NHS) Daten über die medizinische Versorgung ihrer Patienten elektronisch - als anonymisiertes Extrakt aus den Datenbanken ihrer elektronischen Krankengeschichte - zur Verfügung stellten, die Möglichkeit über ein Qualitätsindikatoren-gestütztes Vergütungssystem (Pay for performance, P4P) ein zusätzliches Einkommen zu generieren. Die Einführung der elektronischen Krankengeschichte war somit freiwillig, aber mit der Option eines zusätzlichen Einkommens verbunden. Der Einfluss dieser Massnahme ist unverkennbar: War der Anteil elektronischer Krankengeschichten bis 2004 noch gering, wurde er danach rasant und flächendeckend gesteigert. Bereits im Abrechnungsjahr 2004/2005 nahmen 8486 Hausarztpraxen, welche die Grundversorgung von 99,5\% der Patienten in England verantwortlich sind, die Möglichkeit der P4P-Vergütung wahr und führten demgemäss eine elektronische Krankengeschichte [5]. Diese hohe Teilnahmerate bleibt stabil, wie die aktuellen Daten aus dem Abrechnungsjahr 2010/2011 zeigen [6]. 
Das englische System hat durch die Einführung der P4P-Vergütung gleich mehrfach gewonnen: mehr Transparenz, eine flächendeckende Implementierung der elektronischen Krankengeschichte sowie eine erhebliche Einkommenssteigerung der Hausärzte, die dazu beitrug, den bis dahin herrschenden Nachwuchsmangel von Medizinern in der englischen Grundversorgung zu beheben.

Der englische Weg ist nur einer von vielen möglichen. Ein anderer Ansatz wäre, die vorhandenen Systeme für elektronische Krankengeschichten so weiterzuentwickeln, dass sie für den Hausarzt einen echten Mehrwert in der täglichen Versorgung darstellen. Hier sind insbesondere Applikationen zu nennen, die das Monitoring von chronisch kranken Patienten durch das Aufbereiten der dokumentierten Daten zu medizinische relevanten Informationen ermöglichen. Dass dies gefragt ist, wurde in der vorliegenden Befragung deutlich. Es waren vor allem, die komplexen Softwarefunktionen, die auf einer Datenverarbeitung von klinischen Parametern beruhen (Bsp. Warnhinweis bei kritischer Konstellation von Medikamenten und bestimmten Laborwerten), die von den potenziellen Usern als sehr praxisrelevant eingestuft wurden, wohingegen ein Monitor mit einfacher Zusammenstellung bestimmter Laborparameter ohne Zusatzfunktionen nur von wenigen Teilnehmern als nützlich erachtet wurde. Ebenso riefen vergleichsweise simple Softwareleistungen, wie das Verlinken von Informationsmaterialien innerhalb der eKG, wenig Interesse hervor.

\title{
Limitationen
}

Die Ergebnisse dieser Umfrage sind trotz vergleichsweise hohem Rücklauf mit einer erheblichen Limitation versehen: Eine ausschliessliche Online-Befragung hat einen Selection-Bias, da eher IT-affine Befragte teilnehmen. Dies lässt die Ergebnisse insgesamt noch ernüchternder erscheinen, da unterstellt werden kann, dass die Verbreitung von elektronischen Krankengeschichten in dieser Positivselektion von technikaffinen Ärzten noch höher ist als im Durchschnitt aller Schweizer Ärzte.

\section{Schlussfolgerung}

Die Ergebnisse dieser Querschnittserhebung zeigen eine erhebliche Diskrepanz zwischen den politisch deklarierten eHealth-Zielen und den Grundvoraussetzungen für eine reale Umsetzung in der Schweizer Grundversorgung. Eine sorgfältige Analyse der Implementierungsmöglichkeiten unter Einbezug der unmittelbaren Anwender und ihrer Anforderungen an die Funktionalität von eKG-Softwares scheint dringend angeraten.

\section{Korrespondenzadresse:}

\author{
Dr. med. Sima Djalali \\ Wissenschaftliche Mitarbeiterin \\ Institut für Hausarztmedizin \\ Universitätsspital Zürich \\ Pestalozzistrasse 24 \\ 8091 Zürich \\ sima.djalali@usz.ch
}




\section{Referenzen:}

1. Eidgenössisches Departement des Innern (EDI), Bundesamt für Gesundheit (BAG): Strategie «eHealth» Schweiz. Publikation 27.06.2007; Download am 21.09.2012; http://www.e-healthsuisse.ch/grundlagen/00086/index.html?lang=de

2. Hausärzte Schweiz (MFE): Stellungnahme zum Vorentwurf des Bundesgesetzes über das elektronische Patientendossier (EPDG). Publikation 15.12.2011; Download am 21.09.2012; http://www.hausaerzteschweiz.ch/fileadmin/user_upload/hausaerzteschweiz/Dokumente/Stell ungnahmen/2011-12-19_EPDG_Stellungnahme-MFE.pdf

3. Rosemann T, Marty F, Bhend H, Wagner J, Brunner L, Zoller M: Utilisation of information technologies in ambulatory care in Switzerland. Swiss Med Wkly 2010; 140:w13088.

4. The NHS Information Centre: The Quality and Outcomes Framework (QOF). Download am 21.09.2012; http://www.ic.nhs.uk/statistics-and-data-collections/audits-and-performance/thequality-and-outcomes-framework

5. The NHS Information Centre: The Quality and Outcomes Framework (QOF) 2004/2005. Publikation August 2005; Download 21.09.2012; http://www.ic.nhs.uk/statistics-and-datacollections/supporting-information/audits-and-performance/the-quality-and-outcomesframework/qof-2004/05/qof-2004-05-data-notes

6. The NHS Information Centre: The Quality and Outcomes Framework (QOF) 2010/2011. Publikation Oktober 2011; Download am 21.09.2012; http://www.ic.nhs.uk/statistics-and-datacollections/audits-and-performance/the-quality-and-outcomes-framework/the-quality-andoutcomes-framework-2010-11

7. Online-Umfrage 2010: Download am 10.12.2012; www.praxisinformatik.ch

8. Online-Umfrage 2011: Online-Umfrage 2010: Download am 10.12.2012; www.praxisinformatik.ch 